\title{
Predictive value of thrombus susceptibility for cardioembolic stroke by quantitative susceptibility mapping
}

\author{
Jie Chen ${ }^{1 \# \wedge}$, Zhe Zhang ${ }^{2 \#}$, Ximing $\mathrm{Nie}^{1 \wedge}$, Yuyuan $\mathrm{Xu}^{2}$, Chunlei Liu ${ }^{3}$, Xingquan Zhao ${ }^{1 \wedge}$, Yongjun Wang ${ }^{1,2}$ \\ ${ }^{1}$ Department of Neurology, Beijing Tiantan Hospital, Capital Medical University, Beijing, China; ${ }^{2}$ China National Clinical Research Center for \\ Neurological Diseases, Beijing, China; ${ }^{3}$ Department of Electrical Engineering and Computer Sciences, University of California, Berkeley, CA, USA \\ Contributions: (I) Conception and design: Y Wang, X Zhao, C Liu, J Chen, Z Zhang; (II) Administrative support: Y Wang, X Zhao; (III) Provision \\ of study materials or patients: Y Wang, X Zhao, C Liu; (IV) Collection and assembly of data: J Chen, Z Zhang, Y Xu, X Nie; (V) Data analysis and \\ interpretation: J Chen, Z Zhang, C Liu, Y Wang, N Nie, Y Xu; (VI) Manuscript writing: All authors; (VII) Final approval of manuscript: All authors.
}

\#These authors contributed equally to this work.

Correspondence to: Prof. Yongjun Wang, MD. Department of Neurology, Beijing Tiantan Hospital, Capital Medical University, No 119 S 4th Ring W Rd, Fengtai District, Beijing 100070, China. Email: yongjunwang@ncrcnd.org.cn; Xingquan Zhao, MD. Capital Medical University, No. 119 S 4th Ring W Rd, Fengtai District, Beijing 100070, China. Email: zxq@vip.163.com.

Background: The hypointense blooming signal of thrombi on susceptibility-weighted imaging (SWI), known as the susceptibility vessel sign (SVS), is predictive of cardioembolic stroke. The SVS originates from the local magnetic susceptibility effect; thus, the susceptibility value of thrombi may provide useful information in discriminating stroke etiology. We aim to utilize quantitative susceptibility mapping (QSM) to assess thrombus's susceptibility value in acute ischemic stroke patients and explore the relationship of thrombus susceptibility with cardioembolic stroke.

Methods: From 2018 to 2020, 132 consecutive acute ischemic stroke patients with middle cerebral artery occlusion were recruited within 48 hours of onset. All patients underwent a three-dimensional multi-echo SWI scan using a 3 Tesla magnetic resonance imaging scanner. The SVS presence and the diameter of the SVS-related hypointense signal were assessed on SWI. QSM was applied to compute the susceptibility value of the thrombus. The receiver operating characteristic (ROC) methodology was used to define the optimal cutoff value of the susceptibility in QSM and the diameter on SWI for predicting cardioembolic stroke.

Results: The SVS was identified in 93 (70.5\%) patients with symptomatic middle cerebral artery occlusion and was significantly associated with cardioembolism. The hyperintense signal on QSM in the corresponding middle cerebral artery occlusion was present in $116(87.9 \%)$ patients. ROC analysis indicated that thrombus susceptibility had a greater area under the curve than that of the SVS diameter $(0.88 v s .0 .70$, $\mathrm{P}<0.001)$ and that the optimal cutoff value of thrombus susceptibility for cardioembolism was $0.35 \mathrm{ppm}$. Multivariate analysis demonstrated that thrombus susceptibility $(\geq 0.35 \mathrm{ppm})$ was an independent predictor of cardioembolic stroke (odds ratio $=20.75 ; 95 \% \mathrm{CI}, 7.19-59.87 ; \mathrm{P}<0.001$ ), with sensitivity, specificity, a positive predictive value, and a negative predictive value of $85.2 \%, 80.8 \%, 75.4 \%$, and $88.7 \%$, respectively, while the SVS presence showed sensitivity, specificity, a positive predictive value, and a negative predictive value of $90.7 \%, 43.6 \%, 87.2 \%$, and $52.7 \%$, respectively.

Conclusions: Thrombus susceptibility provides superior diagnostic performance over the SVS for discriminating between cardioembolism and other stroke subtypes. Quantitative susceptibility measurements of thrombi may help predict cardioembolic stroke in patients with acute middle cerebral artery occlusion.

^ ORCID: Jie Chen, 0000-0002-4008-8970; Zhe Zhang, 0000-0001-8220-8443; Ximing Nie, 0000-0002-8380-4076; Xingquan Zhao, 0000-0001-8345-5147. 
Keywords: Susceptibility vessel sign (SVS); quantitative susceptibility mapping (QSM); magnetic resonance imaging (MRI); cardioembolic stroke; acute ischemic stroke

Submitted Mar 02, 2021. Accepted for publication May 20, 2021.

doi: $10.21037 /$ qims-21-235

View this article at: https://dx.doi.org/10.21037/qims-21-235

\section{Introduction}

Cardioembolic stroke is a common but sometimes misdiagnosed cause of acute ischemic stroke. Reliable and straightforward biomarkers of cardioembolic stroke are crucial in clinical practice.

Arterial and cardiac thrombi are distinct in content and structure. Arterial thrombi are rich in platelets, while cardiac thrombi are rich in erythrocytes and fibrin (1). When oxyhemoglobin in erythrocytes is converted to deoxyhemoglobin, methemoglobin, and hemosiderin, the increased paramagnetic susceptibility enhances the blooming hypointense signal known as the susceptibility vessel sign (SVS), on T2*-weighted and susceptibility-weighted imaging (SWI) in magnetic resonance imaging (MRI) (2-5). Many studies have demonstrated that the SVS is correlated with cardioembolic stroke (2-4). Moreover, empirical evidence has shown that several detailed SVS characteristics, such as two-layered SVS, SVS width or diameter, and overestimation ratio, are valuable in predicting cardioembolic stroke (5-8). These characteristics affect varied local susceptibility, indicating that quantitative evaluation of susceptibility can be informative in characterizing thrombus properties and discriminating stroke subtypes. Quantitative susceptibility mapping (QSM) is a potential approach for this evaluation by quantifying the paramagnetic contents in thrombi (9-14). QSM can map the underlying susceptibility of each voxel as a scalar quantity based on gradientrecalled echo (GRE) phase images and has proved valuable in studying iron distribution in neurodegenerative diseases, demyelination in multiple sclerosis, microbleed burden, and cerebral perfusion-related oxygen extraction fraction (13,15-20).

In this study, we aimed to utilize QSM to assess thrombus's susceptibility value in acute ischemic stroke patients and explore the relationship of thrombus susceptibility with cardioembolic stroke.

\section{Methods}

\section{Study participants}

Stroke patients were consecutively enrolled from November
2018 to February 2020 in the present study with the following inclusion criteria: (I) age $\geq 18$ years; (II) acute ischemic stroke confirmed by magnetic resonance angiography (MRA) with documented middle cerebral artery (MCA) occlusion; (III) time from onset to MRI scan less than 48 hours.

The study was conducted following the Declaration of Helsinki (as revised in 2013). This study was approved by the ethics committee of Beijing Tiantan Hospital, and written informed consent was obtained from all participants (or guardians of the participants).

\section{Clinical assessments}

In addition to MRI studies, transthoracic echocardiography, 24-hour electrocardiography (ECG) monitoring, serum B-type natriuretic peptide, complete blood count, chemistry, and coagulation tests were performed on all recruited patients. Ischemic stroke etiology was determined by two neurologists who were blinded to the QSM findings. The TOAST (Trial of Org 10172 in Acute Stroke Treatment) classification system was used to diagnose ischemic stroke subtypes. There are five categories: largeartery atherosclerosis, cardioembolism, small-artery occlusion, a stroke of other determined etiology, and stroke of undetermined etiology (21).

\section{MRI protocol}

All MR images were acquired with a 3.0T MRI scanner (Philips Ingenia, Best, the Netherlands) using a 32-channel head coil. The MRI protocol included 2D diffusionweighted imaging, 2D T2-weighted and 3D T1-weighted structural imaging, 3D fluid-attenuated inversion recovery imaging, 3D time-of-flight MRA, and 3D multi-echo SWI. The 3D SWI acquisition used a flow-compensated 3D 4-echo GRE sequence with TE1 $=6 \mathrm{~ms}$, delta TE $=6.7$ $\mathrm{ms}, \mathrm{TR}=30 \mathrm{~ms}$, flip angle $=15^{\circ}, \mathrm{FOV}=230 \times 230 \times 140 \mathrm{~mm}^{3}$, acquisition voxel size $=0.6 \times 0.6 \times 1.5 \mathrm{~mm}^{3}$, and reconstruction voxel size $=0.48 \times 0.48 \times 0.75 \mathrm{~mm}^{3}$. The raw magnitude and phase images of all echoes were saved for QSM calculation. 

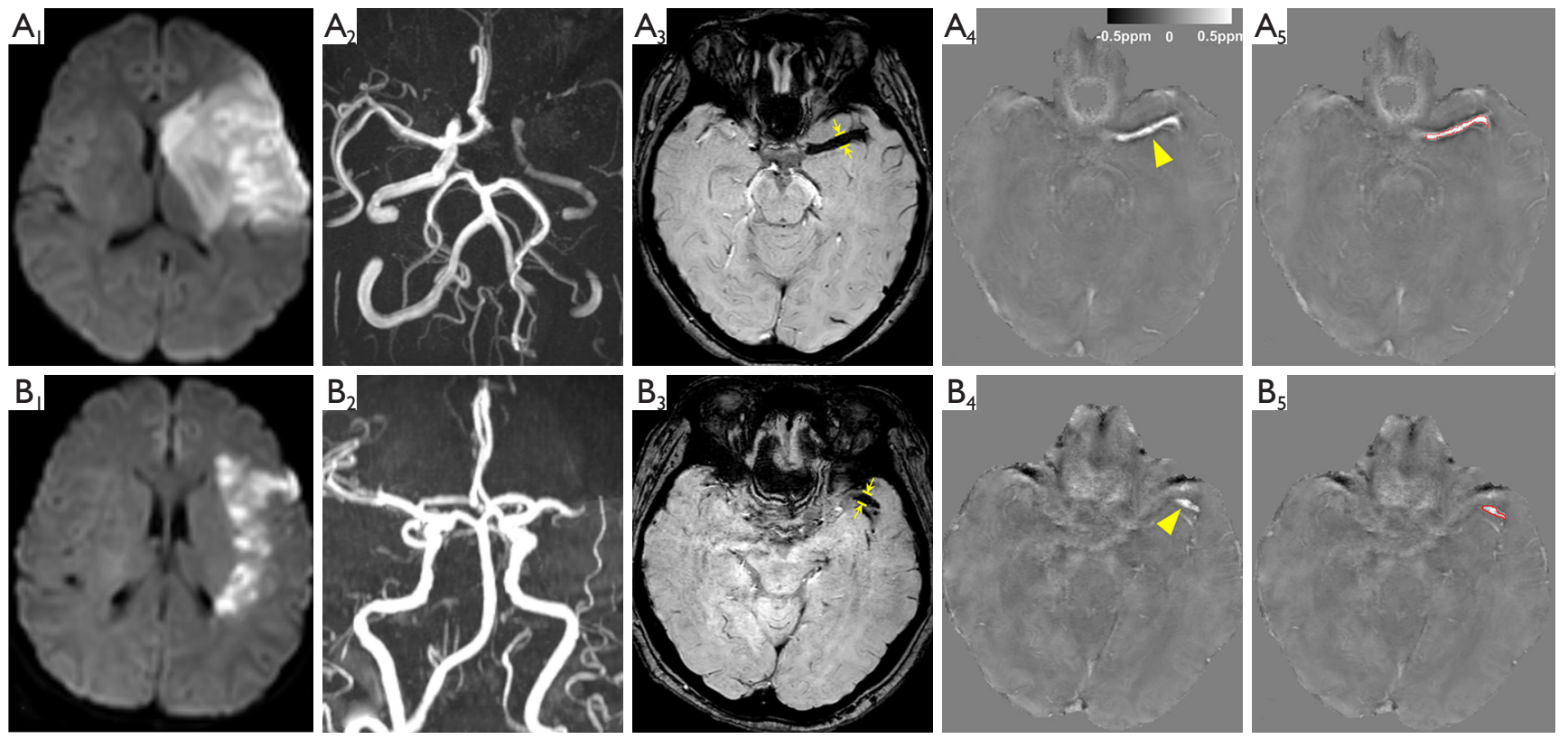

Figure 1 A demonstration of thrombi measurements on SWI and QSM images. (A) A 25-year-old female patient with cardioembolic stroke imaged 3 hours following the onset of right hemiparesis and aphasia. DWI demonstrates acute left MCA infarctions (A1), accompanied by MCA occlusion on MRA (A2). SWI showed an SVS with a diameter of $5.62 \mathrm{~mm}$ in the occluded vessel (A3, yellow arrow). QSM maps show a hyperintense signal in the thrombus (A4, yellow arrowhead), with a mean susceptibility value of 0.455 ppm measured in the ROI (A5, in red contour). (B) A 50-year-old female patient with right hemiparesis and aphasia; her stroke subtype is classified as large artery atherosclerosis. There are left hemisphere infarctions on DWI (B1), left MCA occlusion on MRA (B2), and an SVS with a diameter of 4.38 $\mathrm{mm}$ on SWI (B3, yellow arrow). The signal in the thrombus on QSM maps is low (B4, yellow arrowhead), with a mean susceptibility value of $0.231 \mathrm{ppm}$ measured in the ROI (B5, in red contour). DWI, diffusion-weighted imaging; MCA, middle cerebral artery; MRA, magnetic resonance angiography; SWI, susceptibility-weighted imaging; SVS, susceptibility vessel sign; QSM, quantitative susceptibility mapping; ROI, region of interest.

\section{SVS measurement}

Two investigators performed the SVS measurement, and QSM region of interest (ROI) analyses (JC and $\mathrm{XMN}$ ) blinded to the clinical data. The SVS was defined as a hypointense signal on SWI in the corresponding symptomatic occlusive vessels, and the diameter of the vessels should exceed that of the contralateral vessels (2). The diameter of the SVS-related hypointense signal was obtained by measuring the maximum radial length of the hypointense vessel on SWI (6).

\section{QSM calculation}

The quantitative susceptibility maps were calculated using the STI Suite v2.2 toolbox (22) in MATLAB (Mathworks, Natick, MA, USA). The binary brain mask was generated from the magnitude image of the 1 st echo using the brain extraction tool provided by FSL (FMRIB, Oxford University, UK) (23). The phase images from the multiecho acquisition were processed using Laplacian-based phase unwrapping, variable-kernel sophisticated harmonic artifact reduction for phase data (known as V-SHARP) for background phase removal, and iterative LSQR QSM $(24,25)$. The quantitative susceptibility maps were saved in a nifti-1 (Neuroimaging Informatics Technology Initiative, https://nifti.nimh.nih.gov/nifti-1/) format and loaded in a 3D slicer for ROI drawing and ROI statistics (26). The ROI for susceptibility measurement was drawn on the central axial slice of the hyperintense thrombus in the corresponding symptomatic occlusive vessels using the $3 \mathrm{D}$ slicer segmentation module (Figure 1) (27). The thrombus susceptibility value [in units of parts per million (ppm)] and thrombus volume of the ROIs were extracted using the 3D slicer quantification module. 


\section{Statistical analysis}

Continuous variables are presented as mean \pm standard deviation or median, and categorical variables are presented as percentages. The Student's $t$-test and Mann-Whitney $\mathrm{U}$ test were performed on continuous variables; Pearson's $\chi^{2}$ test was used to compare categorical variables between groups. Spearman's method was used to analyze the correlation between the diameter of the hypointense signal on SWI and the susceptibility of the hyperintense signal on QSM. The receiver operating characteristic (ROC) methodology was used to define the optimal cutoff value and compare the SVS diameter and thrombus susceptibility in predicting cardioembolic stroke. We calculated the diagnostic values [sensitivity, specificity, positive predictive value (PPV), negative predictive value (NPV), and 95\% confidence interval (CI) for diagnosing cardioembolism based on the SVS, diameter, and susceptibility. To control for potential confounders of cardioembolic stroke, multivariate logistic regression analysis was utilized to examine the association between susceptibility and cardioembolism, where age, sex, National Institutes of Health Stroke Scale (NIHSS) score, time to MR scan, and MCA branch occlusion were adjusted. The Kendall $\mathrm{W}$ test was used to measure interobserver agreement concerning the SVS diameter and QSM values. Statistical significance was set at $\mathrm{P}<0.05$. Statistical analyses were performed using SPSS Version 24 (IBM, SPSS; Chicago, IL), except that the ROC analysis was performed using MedCalc V.19.1 (MedCalc Software, Belgium).

\section{Results}

A total of 153 patients were registered during the study period. Of these, 21 patients were excluded because of severe image artifacts $(n=15)$ or incomplete clinical data $(n=6)$. Therefore, 132 patients were included in the final analysis.

The participants were 86 males $(65.2 \%)$ and 46 females $(34.8 \%)$ with a mean age of $62.15 \pm 12.67$ years (range, 25-87 years). The median baseline NIHSS score was 13 (interquartile range, 10-17). The mean time from stroke onset to the MR scan was $11.02 \pm 10.80$ hours (range, 0.85-48 hours). Intravenous alteplase treatment (IVT) was administered to 61 patients (46.2\%) before the MRI scan. The most common etiology of ischemic stroke was large artery atherosclerosis in 67 of the participants (50.8\%), followed by cardioembolism in 54 (40.9\%), undetermined etiologies in $6(4.5 \%)$, and other etiologies (including cerebral vasculitis, dissection, and hypercoagulable state) in $5(3.8 \%)$. Compared with the noncardioembolism group, patients with cardioembolism were older, with a higher proportion of females, higher NIHSS scores, and a shorter time between stroke onset and the MR scan distribution of IVT was similar between groups. Baseline characteristics are summarized in Table 1.

The SVS was present in $93(70.5 \%)$ patients with symptomatic MCA occlusion, which was more frequent among patients with cardioembolism than those with noncardioembolism (Table 1).

The hyperintense signal on QSM in the corresponding MCA occlusion was present in 116 (87.9\%) patients. The susceptibility of the hyperintense thrombus on QSM and the diameter of the hypointense SVS on SWI were measured (Figure 1). The interobserver agreement of the mean thrombus susceptibility value measurement and SVS diameter measurement by the Kendall $W$ value were 0.57 and 0.6 , respectively. The susceptibility of thrombus was significantly higher in patients with cardioembolic stroke than other stroke subtypes $(0.44 \pm 0.09 \mathrm{ppm}$ versus $0.28 \pm 0.11 \mathrm{ppm}, \mathrm{P}<0.001)$. The thrombus susceptibility was significantly correlated with the SVS diameter, with a correlation coefficient of $0.55(\mathrm{P}<0.001)$.

ROC curve analysis was performed to investigate the diagnostic efficiency of the thrombus susceptibility and the SVS diameter in predicting cardioembolic stroke. The susceptibility ROC area under the curve $(0.88)$ was greater than that of the diameter $(0.70, \mathrm{P}<0.001$, Figure 2$)$. The optimal cutoff value for thrombus susceptibility and the SVS diameter for cardioembolism was $0.35 \mathrm{ppm}$ and $4.1 \mathrm{~mm}$, respectively. In this setting, QSM susceptibility $(\geq 0.35 \mathrm{ppm}$ ) yielded a sensitivity, specificity, PPV, and NPV of $85.2 \%, 80.8 \%, 75.4 \%$, and $88.7 \%$, respectively, for cardioembolic stroke and was superior to the SVS for cardioembolism identification (Table 2).

Multivariate analysis revealed that SVS susceptibility $(\geq 0.35 \mathrm{ppm})$ was independently associated with cardioembolism after controlling for the effects of age, sex, NIHSS score, the time between onset to the MR scan, and site of occlusion (Table 3; odds ratio, 20.75; 95\% CI, 7.1959.87; $\mathrm{P}<0.001)$.

\section{Discussion}

The present study demonstrated that the magnetic susceptibility of the thrombus in occluded MCA can be 
Table 1 Baseline characteristics

\begin{tabular}{|c|c|c|c|}
\hline Characteristics & CE $(n=54)$ & NonCE $(n=78)$ & $P$ value \\
\hline Sex (male), n (\%) & $28(50.9)$ & $58(74.4)$ & 0.008 \\
\hline NIHSS, median (IQR) & 14.5 (12 to 19$)$ & 12 (8 to 15.25$)$ & 0.000 \\
\hline Atrial fibrillation, n (\%) & $50(92.6)$ & $0(0.0)$ & 0.000 \\
\hline Hypertension, n (\%) & $31(57.4)$ & $53(67.9)$ & 0.270 \\
\hline Diabetes, n (\%) & $13(24.1)$ & $19(24.4)$ & 0.970 \\
\hline Hyperlipidemia, n (\%) & $16(29.6)$ & $34(43.6)$ & 0.194 \\
\hline Current smoking, n (\%) & $24(44.4)$ & $48(61.5)$ & 0.052 \\
\hline Site of occlusion, n (\%) & & & 0.049 \\
\hline MCA-M1 & $38(70.4)$ & $66(84.6)$ & \\
\hline MCA-M2 & $16(29.6)$ & $12(15.4)$ & \\
\hline SVS, n (\%) & $49(90.7)$ & $44(56.4)$ & 0.000 \\
\hline
\end{tabular}

CE, cardioembolism; MCA, middle cerebral artery; SVS, susceptibility vessel sign; MR, magnetic resonance; NIHSS, National Institutes of Health Stroke Scale; nonCE, noncardioembolism; SD, standard deviation; IQR, interquartile range.

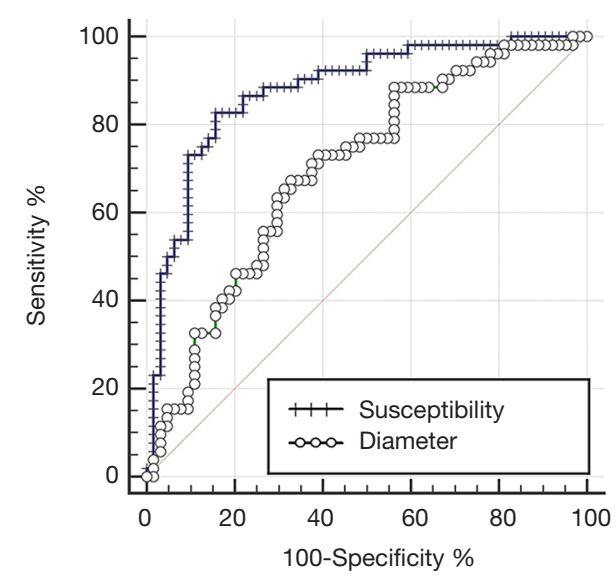

Figure 2 Comparison of ROC curves of thrombus susceptibility to SVS diameter for predicting cardioembolic stroke. ROC, receiver operating characteristic; SVS, susceptibility vessel sign.

quantified using QSM and is significantly associated with cardioembolic stroke. The susceptibility value could more accurately discriminate cardioembolic stroke from other stroke etiologies than the SVS characteristics on SWI.
The current study showed that the SVS on SWI was a sensitive predictor of cardioembolic stroke, with a sensitivity of $90.7 \%$. It was consistent with many previous studies, which demonstrated the SVS and susceptibility property value for predicting stroke etiology and vessel recanalization (2-4,6-8). The SVS originates from the local magnetic susceptibility increase; the correlation between increased susceptibility and cardioembolism is based on the theory that cardiac thrombi (usually due to atrial fibrillation, then left ventricular dysfunction, followed by blood stasis) are rich in erythrocytes and fibrin, while arterial thrombi (typically associated with atherosclerotic plaque rupture that triggers the aggregation of platelets) are rich in platelets (28). The deoxyhemoglobin and methemoglobin in erythrocytes are all paramagnetic materials, leading to the increased magnetic susceptibility of cardiac thrombi compared to arterial sources. By taking advantage of the paramagnetic property of deoxyhemoglobin, SWI enhances the visualization of the thrombus with the blooming hypointense signal in the corresponding symptomatic occlusive vessels (13).

However, SWI fundamentally suffers from the inherent blooming effect (e.g., sometimes it cannot distinguish 
Table 2 Diagnostic accuracy of thrombus image characteristics for cardioembolic stroke

\begin{tabular}{|c|c|c|c|c|c|c|c|c|}
\hline Thrombus image characteristics- & \multicolumn{2}{|c|}{ Sensitivity } & \multicolumn{2}{|c|}{ Specificity } & \multicolumn{2}{|c|}{ PPV } & \multicolumn{2}{|c|}{ NPV } \\
\hline SVS & 90.7 & $78.9-96.5$ & 43.6 & $32.3-55.3$ & 87.2 & $74.0-94.2$ & 52.7 & $47.4-58.0$ \\
\hline Diameter (4.1 mm) & 59.3 & $45.8-71.9$ & 74.0 & $62.4-83.6$ & 64.8 & $54.3-74.1$ & 69.2 & $61.6-75.9$ \\
\hline Susceptibility (0.35 ppm) & 85.2 & $72.9-93.4$ & 80.8 & $70.3-88.8$ & 75.4 & $65.8-83.0$ & 88.7 & $80.5-93.8$ \\
\hline
\end{tabular}

$\mathrm{Cl}$, confidence interval; NPV, negative predictive value; PPV, positive predictive value; SVS, susceptibility vessel sign.

Table 3 Predictors of cardioembolic stroke based on multiple logistic regression analysis

\begin{tabular}{lccc}
\hline Variable & OR & $95 \% \mathrm{Cl}$ & $\mathrm{P}$ value \\
\hline Susceptibility $\geq 0.35 \mathrm{ppm}$ & 20.75 & $7.19-59.87$ & 0.000 \\
Age, years & 0.99 & $0.95-1.03$ & 0.536 \\
Male sex & 1.88 & $0.67-5.35$ & 0.234 \\
NIHSS & 0.89 & $0.82-0.97$ & 0.008 \\
Time to MR & 1.00 & $0.95-1.05$ & 0.950 \\
MCA branch occlusion & 1.91 & $0.57-6.43$ & 0.295 \\
\hline
\end{tabular}

Variables were selected for entry into the models based on the results of univariate analysis $(P \leq 0.05)$. The Hosmer-Lemeshow goodness-of-fit showed $\chi^{2}=8.83$, and $\mathrm{P}=0.357$, demonstrating a good fit of the models. OR, odds ratio; $\mathrm{Cl}$, confidence interval; NIHSS, National Institutes of Health Stroke Scale; MR, magnetic resonance; MCA, middle cerebral artery.

the SVS from the background or cannot observe the susceptibility distribution in bulk hypointense signals). QSM, on the other hand, is insensitive to the scan parameter and tissue-susceptibility-dependent blooming artifacts and offers a quantitative measurement of tissue susceptibility (13). This study confirmed that the magnetic susceptibility of the thrombus was positively correlated with the SVS diameter. Moreover, the thrombus susceptibility value showed higher accuracy than the SVS diameter for predicting cardioembolic stroke, indicating that thrombus susceptibility can be used as an imaging biomarker of cardioembolism in acute ischemic stroke. Similar findings have been reported in intracranial hemorrhage studies, in which susceptibility values showed improved accuracy in assessing both hematoma and microbleed burden $(17,29)$.

Cardioembolic stroke is easily misdiagnosed in clinical practice. Atrial fibrillation, the most common risk factor for cardioembolism, is frequently paroxysmal and asymptomatic and may evade detection by routine ECG monitoring. This study demonstrated that acute ischemic stroke patients with QSM thrombus susceptibility $\geq 0.35 \mathrm{ppm}$ were more likely to have cardioembolism. Therefore, for an acute ischemic stroke patient with increased susceptibility in occluded MCA but without cardioembolism evidence, prolonged ECG monitoring, echocardiography, and other cardiac examinations should be considered. These cardiac examinations may prompt the discovery of cardioembolic stroke and a change in treatment strategies to prevent future embolic events.

Our study had several limitations. First, it was a singlecenter pilot investigation with relatively small sample size. Some baseline characteristics were not similar between the cardioembolism and noncardioembolism groups. Although logistic regression analysis was used to control these possible confounding factors, the imbalance cannot be ruled out. Second, 61 (46.2\%) patients received IVT before the MRI scan. However, all of the patients recruited in this study had MCA occlusion with blood clots that did not dissolve or break up after IVT. In this setting, fibrin content may have changed with tissue plasminogen activator, but deoxyhemoglobin and hemosiderin content may have not. Thus, susceptibility quantification may not have changed dramatically before the clot was fully dissolved. 


\section{Conclusions}

The susceptibility of the thrombus in the occluded MCA can be quantified using QSM. Thrombus susceptibility $(\geq 0.35 \mathrm{ppm})$ showed a higher diagnostic performance than the SVS for the discrimination between cardioembolism and other stroke subtypes. Susceptibility measurements of thrombi may help predict cardioembolic stroke in patients with acute MCA occlusion.

\section{Acknowledgments}

Funding: This study was supported by the National Natural Science Foundation of China (81701139).

\section{Footnote}

Conflicts of Interest: All authors have completed the ICMJE uniform disclosure form (available at https://dx.doi. org/10.21037/qims-21-235). The authors have no conflicts of interest to declare.

Ethical Statement: The authors are accountable for all aspects of the work in ensuring that questions related to the accuracy or integrity of any part of the work are appropriately investigated and resolved. The study was conducted in accordance with the Declaration of Helsinki (as revised in 2013). This study was approved by the ethics committee of Beijing Tiantan Hospital, and written informed consent was obtained from all participants or guardians of the participants.

Open Access Statement: This is an Open Access article distributed in accordance with the Creative Commons Attribution-NonCommercial-NoDerivs 4.0 International License (CC BY-NC-ND 4.0), which permits the noncommercial replication and distribution of the article with the strict proviso that no changes or edits are made and the original work is properly cited (including links to both the formal publication through the relevant DOI and the license). See: https://creativecommons.org/licenses/by-nc-nd/4.0/.

\section{References}

1. Kamel H, Healey JS. Cardioembolic Stroke. Circ Res 2017;120:514-26.

2. Cho KH, Kim JS, Kwon SU, Cho AH, Kang DW. Significance of susceptibility vessel sign on $\mathrm{T} 2{ }^{*}$-weighted gradient echo imaging for identification of stroke subtypes. Stroke 2005;36:2379-83.

3. Bourcier R, Derraz I, Delasalle B, Beaumont M, Soize S, Legrand L, Desal H, Bracard S, Naggara O, Oppenheim C, THRACE investigators. Susceptibility vessel sign and cardioembolic etiology in the THRACE Trial. Clin Neuroradiol 2019;29:685-92.

4. Liu M, Li L, Li G. The different clinical value of susceptibility vessel sign in acute ischemic stroke patients under different interventional therapy: A systematic review and meta-analysis. J Clin Neurosci 2019;62:72-9.

5. Bourcier R, Derraz I, Bracard S, Oppenheim C, Naggara O, THRACE Investigators. Two-layered susceptibility vessel sign and high overestimation ratio on mri are predictive of cardioembolic stroke. AJNR Am J Neuroradiol 2019;40:65-7.

6. Kang DW, Jeong HG, Kim DY, Yang W, Lee SH. Prediction of stroke subtype and recanalization using susceptibility vessel sign on susceptibility-weighted magnetic resonance imaging. Stroke 2017;48:1554-9.

7. Yamamoto N, Satomi J, Tada Y, Harada M, Izumi Y, Nagahiro S, Kaji R. Two-layered susceptibility vessel sign on 3-tesla $\mathrm{T}^{*}$-weighted imaging is a predictive biomarker of stroke subtype. Stroke 2015;46:269-71.

8. Zhang R, Zhou Y, Liu C, Zhang M, Yan S, Liebeskind DS, Lou M. Overestimation of susceptibility vessel sign: a predictive marker of stroke cause. Stroke 2017;48:1993-6.

9. de Rochefort L, Brown R, Prince MR, Wang Y. Quantitative MR susceptibility mapping using piece-wise constant regularized inversion of the magnetic field. Magn Reson Med 2008;60:1003-9.

10. Haacke EM, Liu S, Buch S, Zheng W, Wu D, Ye Y. Quantitative susceptibility mapping: current status and future directions. Magn Reson Imaging 2015;33:1-25.

11. Shmueli K, de Zwart JA, van Gelderen P, Li TQ, Dodd SJ, Duyn JH. Magnetic susceptibility mapping of brain tissue in vivo using MRI phase data. Magn Reson Med 2009;62:1510-22.

12. Wang Y, Liu T. Quantitative susceptibility mapping (QSM): Decoding MRI data for a tissue magnetic biomarker. Magn Reson Med 2015;73:82-101.

13. Liu C, Li W, Tong KA, Yeom KW, Kuzminski S. Susceptibility-weighted imaging and quantitative susceptibility mapping in the brain. J Magn Reson Imaging 2015;42:23-41.

14. Wei H, Cao S, Zhang Y, Guan X, Yan F, Yeom KW, Liu C. Learning-based single-step quantitative susceptibility mapping reconstruction without brain extraction. 
NeuroImage 2019;202:116064.

15. Kaunzner UW, Kang Y, Zhang S, Morris E, Yao Y, Pandya S, Hurtado Rua SM, Park C, Gillen KM, Nguyen TD, Wang Y, Pitt D, Gauthier SA. Quantitative susceptibility mapping identifies inflammation in a subset of chronic multiple sclerosis lesions. Brain 2019;142:133-45.

16. Kudo K, Liu T, Murakami T, Goodwin J, Uwano I, Yamashita F, Higuchi S, Wang Y, Ogasawara K, Ogawa A, Sasaki M. Oxygen extraction fraction measurement using quantitative susceptibility mapping: Comparison with positron emission tomography. J Cereb Blood Flow Metab 2016;36:1424-33.

17. Liu T, Surapaneni K, Lou M, Cheng L, Spincemaille P, Wang Y. Cerebral microbleeds: burden assessment by using quantitative susceptibility mapping. Radiology 2012;262:269-78.

18. Lu X, Ma Y, Chang EY, He Q, Searleman A, von Drygalski A, Du J. Simultaneous quantitative susceptibility mapping (QSM) and R2* for high iron concentration quantification with $3 \mathrm{D}$ ultrashort echo time sequences: An echo dependence study. Magn Reson Med 2018;79:2315-22.

19. Poston KL, Ua Cruadhlaoich MAI, Santoso LF, Bernstein JD, Liu T, Wang Y, Rutt B, Kerchner GA, Zeineh MM. Substantia nigra volume dissociates bradykinesia and rigidity from tremor in Parkinson's disease: A 7 Tesla imaging study. J Parkinsons Dis 2020;10:591-604.

20. Wei H, Zhang C, Wang T, He N, Li D, Zhang Y, Liu C, Yan F, Sun B. Precise targeting of the globus pallidus internus with quantitative susceptibility mapping for deep brain stimulation surgery. J Neurosurg 2019. doi: 10.3171/2019.7.JNS191254. [Epub ahead of print].

21. Adams HP, Bendixen BH, Kappelle LJ, Biller J, Love BB, Gordon DL, Marsh EE. Classification of subtype of acute ischemic stroke. Definitions for use in a multicenter clinical trial. TOAST. Trial of Org 10172 in Acute Stroke Treatment. Stroke 1993;24:35-41.

22. Li W, Avram AV, Wu B, Xiao X, Liu C. Integrated Laplacian-based phase unwrapping and background phase removal for quantitative susceptibility mapping. NMR Biomed 2014;27:219-27.

23. Smith SM. Fast robust automated brain extraction. Hum Brain Mapp 2002;17:143-55.

24. Li W, Wang N, Yu F, Han H, Cao W, Romero R, Tantiwongkosi B, Duong TQ, Liu C. A method for estimating and removing streaking artifacts in quantitative susceptibility mapping. Neuroimage 2015;108:111-22.

25. Wu B, Li W, Guidon A, Liu C. Whole brain susceptibility mapping using compressed sensing. Magn Reson Med 2012;67:137-47.

26. Fedorov A, Beichel R, Kalpathy-Cramer J, Finet J, FillionRobin JC, Pujol S., Bauer C., Jennings D, Fennessy F, Sonka M, Buatti J, Aylward S, Miller JV, Pieper S., Kikinis R. 3D Slicer as an image computing platform for the Quantitative Imaging Network. Magn Reson Imaging 2012;30:1323-41.

27. Zheng MZ, Yang QY, Lu XD, Hu SL, Chai C, Shen W, Chang BG, Wang ZY, Xia S. Middle cerebral artery thrombus susceptibility-weighted imaging mapping predicts prognosis. Quant Imaging Med Surg 2019;9:1556-65.

28. Byrnes JR, Wolberg AS. Red blood cells in thrombosis. Blood 2017;130:1795-9.

29. Zhang Y, Wei H, Sun Y, Cronin MJ, He N, Xu J, Zhou Y, Liu C. Quantitative susceptibility mapping (QSM) as a means to monitor cerebral hematoma treatment. J Magn Reson Imaging 2018;48:907-15.
Cite this article as: Chen J, Zhang Z, Nie X, Xu Y, Liu C, Zhao X, Wang Y. Predictive value of thrombus susceptibility for cardioembolic stroke by quantitative susceptibility mapping. Quant Imaging Med Surg 2022;12(1):550-557. doi: 10.21037/ qims-21-235 